\title{
Current concepts in the surgical management of lagophthalmos in leprosy
}

\author{
PAUL COURTRIGHT* \& SUSAN LEWALLEN $\dagger$ \\ *Department of Ophthalmology, University of British Columbia, St \\ Paul's Hospital, 1081 Burrard Street, Vancouver, BC V6Z 1 Y6 \\ Canada; and $\dagger 3720$ Harrison Street, Bellingham, WA 98226, USA
}

\author{
Accepted for publication 23 March 1995
}

\begin{abstract}
Summary Existing data suggest that, at a minimum, $2 \%$ of paucibacillary patients and $5 \%$ of multibacillary patients have lagophthalmos; at least 290,000 people worldwide have leprosy-related lagophthalmos. Surgical intervention is the only method for correcting lagophthalmos; effectiveness of the different procedures commonly used has not been measured. Results from a survey of eye care providers revealed that surgeons in Asia used a wide range of different techniques for the correction of lagophthalmos while almost all of the surgeons in Africa used tarsorrhaphy. There is a need to evaluate surgical outcome of these techniques and to develop guidelines to assist in increasing the number of surgeries for lagophthalmos in leprosy patients.
\end{abstract}

\section{Introduction}

Tremendous strides in multidrug therapy (MDT) coverage have been made over the past two decades; in 1992 it was estimated that about $55 \%$ of registered leprosy patients were under MDT. ${ }^{1}$ Deformity prevention and management (particularly related to ocular disease) have been more difficult to achieve.

Estimating the number of leprosy patients with lagophthalmos is problematic. Nevertheless, existing data suggest that, at a minimum, $2 \%$ of paucibacillary patients ${ }^{2}$ and $5 \%$ of multibacillary patients ${ }^{3}$ have lagophthalmos. Using estimates of total leprosy patients (including those released from treatment) it can be calculated that at least 290,000 people worldwide have leprosy-related lagophthalmos. Lagophthalmos is a serious complication of leprosy; corneal disease and vision loss are common. The stigmatizing characteristics of lagophthalmos are also significant. Prevention, the purview of general medical/paramedical personnel, should be the primary method for controlling lagophthalmos. For paucibacillary patients the early recognition of facial patches and adequate steroid treatment of reversal reactions ${ }^{4}$ are the most effective tools in the prevention of lagophthalmos. In multibacillary disease early and adequate antileprosy therapy will prevent lagophthalmos from developing in many cases. ${ }^{3}$ 
However, until these measures are achieved and all patients are on MDT, lagophthalmos will continue to be a significant cause of ocular morbidity in leprosy.

Surgical intervention is the only method for correcting lagophthalmos and preventing corneal disease and vision loss. Recommendations for surgical intervention are not straightforward. There are a number of different surgical procedures for the correction of lagophthalmos and there is conflicting evidence on their effectiveness; these must also be evaluated in the context of available resources.

In a review of 33 temporalis transposition surgeries (in 22 patients) in Pakistan Weber et al., report that $51 \cdot 5 \%$ did not have a 'good result' based on the physical findings and patients' opinion. ${ }^{5}$ Betharia and Bhaumik in India reviewed the outcome of 10 eyes with surgical correction by a modified Kuhnt Szymanowski operation and 10 eyes with a facial sling. ${ }^{6}$ In both groups 8 or more patients had cosmetic improvement; further evaluation is impossible due to the small number of patients. An evaluation of the sling procedure in 54 eyes in China demonstrated 'good' or 'excellent' results in $63 \%$ of cases after a 2-6 month postoperative follow up (Lu Bin-xin, personal communication).

We sought to determine the frequency of use of common surgical procedures for lagophthalmos among ophthalmologists and other eye care providers treating leprosy patients, and the usual criteria used for the selection of patients for surgery.

\section{Materials and methods}

A questionnaire was distributed (through ILEP, Christoffel Blindenmission and by direct mail) to leprosy ophthalmologists and leprosy institutions worldwide. The questionnaire included questions related to patient population, number of surgeries performed, surgical procedure(s) used, criteria for selection of patients for surgery, and criteria for selection of surgical procedures.

There have been a profusion of names applied to surgical procedures for lagophthalmos which work on the same principle, but differ in slight ways. For our review we tried to classify all procedures into one of four groups which included:

1 Temporalis transfer. A part of the temporalis muscle is transferred to the eyelids so that use of the jaw causes the lids to close. This complex procedure requires physical therapy for success and has a good cosmetic result.

2 Tarsorrhaphy. This includes any method which permanently closes part of the eyelid margin temporally and sometimes nasally as well. This simple procedure does not require physical therapy for success but often does not result in a good cosmetic effect.

3 Lid suspension. This refers to any operation in which some material, e.g., nylon suture, fascia lata, is placed on the lid margin in order to raise the lid margin by resuspending it. This procedure is of moderate complexity, requiring no physical therapy for success and has a good cosmetic effect.

4 Horizontal shortening. This refers to any one of the many procedures which works by removing a piece of lid (shortening the lid in the horizontal direction) usually at the lateral canthal angle. Modifications are referred to as the tarsal strip, modified Kuhnt Szymanowski, Fox, or Bick procedure. This procedure is of moderate complexity, requiring no physical therapy for success and has a good cosmetic effect. 
Table 1. Common surgical procedures used for the correction of lagophthalmos: frequency of reporting

\begin{tabular}{lccr}
\hline & \multicolumn{3}{c}{ Reported use } \\
\cline { 2 - 4 } & $\begin{array}{c}\text { Asia } \\
(n=14)\end{array}$ & $\begin{array}{c}\text { Africa } \\
(n=8)\end{array}$ & $\begin{array}{r}\text { Overall } \\
(n=27)\end{array}$ \\
Surgical procedure & $64 \cdot 3 \%$ & $0 \%$ & $42 \cdot 3 \%$ \\
Horizontal shortening & $57 \cdot 1 \%$ & $0 \%$ & $38 \cdot 5 \%$ \\
Temporalis transfer & $64 \cdot 3 \%$ & $100 \%$ & $76 \cdot 9 \%$ \\
Tarsorrhaphy & $14.3 \%$ & $0 \%$ & $7 \cdot 7 \%$ \\
Lid suspension & & & \\
\hline
\end{tabular}

\section{Results}

Twenty-seven completed forms were received; most were from clinicians working in Asia or Africa. Overall, the median proportion of leprosy patients undergoing surgical correction of lagophthalmos in the sites was $0 \cdot 2 \%$. The most commonly reported procedure used for correction of lagophthalmos was tarsorrhaphy (Table 1). Surgeons in Africa relied on this procedure almost exclusively while surgeons in Asia reported using tarsorrhaphy, horizontal shortening and TMT almost equally. Degree of corneal exposure, corneal damage, and ectropion (but not corneal hypesthesia) were commonly reported indications for surgery (Table 2).

\section{Discussion}

The best surgical procedure for the correction of lagophthalmos would have the following characteristics:

1 It could be performed by a trained health worker in the field instead of by an ophthalmologist in a hospital.

2 It would require minimal instruments and supplies and little time to perform.

3 It would not require any preoperative or postoperative physical therapy.

4 It would give a good cosmetic result and lead to high patient satisfaction.

5 It would give a long-term solution, not requiring any additional surgery.

6 It would prevent the development of corneal damage due to corneal exposure.

Clearly, none of the commonly-used surgeries for lagophthalmos fit all of these

Table 2. Criteria for the selection of patients for lagophthalmos surgery

\begin{tabular}{lc} 
Criteria & $\%$ of respondents reporting* \\
\hline Exposure of cornea & $72 \%$ \\
Ectropion & $64 \%$ \\
Corneal damage & $72 \%$ \\
Corneal hypesthesia & $28 \%$ \\
\hline
\end{tabular}

* Multiple criteria could be selected by respondents. 
characteristics; this explains some of the wide variation in use of different procedures worldwide. Interpretation of our results is limited by the lack of objective criteria for measuring success and failure with surgical correction. We do not claim to have a representative sample of clinicians; surgeons who returned the questionnaire were interested in the topic. Nevertheless, the findings from this survey and recent publications suggest that there is a need for evaluating surgical correction of lagophthalmos in leprosy. There is a need to measure the outcome of tarsorrhaphy and horizontal shortening in the hands of health workers under routine field conditions. The high proportion of TMT patients with a poor result in Pakistan (and anecdotal reports elsewhere) suggests that a scientific evaluation of TMT patients in other settings is needed as soon as possible. If TMT does not offer adequate long-term results in these patients it should be discontinued. Objective criteria for targeting patients in need of surgery are needed. Training programmes for health workers need to be developed and standardized. Educational programmes aimed at motivating patients to demand surgery need to be developed. These activities should assist health workers in reducing ocular disability due to lagophthalmos.

\section{Acknowledgments}

We would like to thank ILEP, Christoffel Blindenmission and the respondents for their cooperation in this survey.

\section{References}

${ }^{1}$ Pathmanathan I. The role of health systems research in leprosy control. Lepr Rev 1992; 63(Suppl): 106s$113 \mathrm{~s}$.

2 Hogeweg M, Kiran KU, Suneetha S. The significance of facial patches and Type 1 reaction for the development of facial nerve damage in leprosy. A retrospective study among 1226 paucibacillary leprosy patients. Lepr Rev 1991; 62: 143-149.

3 Courtright P, Lewallen S, Li HY, Hu LF. Lagophthalmos in a multibacillary leprosy population under multidrug therapy in the People's Republic of China. Le pr Rev 1996; 66, 214-219.

4 Kiran KU, Hogeweg M, Suneetha S. Treatment of recent facial nerve damage with lagophthalmos, using a semistandardized steroid regimen. Lepr Rev 1991; 62: 150-154.

5 Weber MW, Van Soest A, Neff G, Chiang T, Pfau R. Results of surgical procedures for the correction of foot-drop and of lagophthalmos due to leprosy. Lepr Rev 1992; 63: 255-262.

6 Betharia SM, Bhaumik S. Evaluation of different surgical techniques in the management of paralytic ectropion in cases of leprosy. Afro-Asian J Ophthalmol 1989; 7: 121-124. 